\title{
Overexpression of lipoprotein lipase improves insulin resistance induced by a high-fat diet in transgenic rabbits
}

\author{
S. Kitajima ${ }^{1} \cdot$ M. Morimoto ${ }^{1}$ E. Liu ${ }^{1}$ T. Koike ${ }^{2}$ Y. Higaki ${ }^{3}$ Y. Taura ${ }^{4} \cdot$ K. Mamba M K. Itamoto $^{4} \cdot$ \\ T. Watanabe 6 - K. Tsutsumi ${ }^{7} \cdot$ N. Yamada $^{8} \cdot$ J. Fan $^{2}$ \\ ${ }^{1}$ Analytical Research Center for Experimental Sciences, Saga Medical School, Saga, Japan \\ ${ }^{2}$ Cardiovascular Disease Laboratory, Department of Pathology, Institute of Basic Medical Sciences, University of Tsukuba, \\ Tsukuba, Japan \\ ${ }^{3}$ Department of Preventive Medicine, Faculty of Medicine, Saga University, Saga, Japan \\ ${ }^{4}$ Department of Veterinary Surgery, Veterinary Clinical Science, Yamaguchi University, Yamaguchi, Japan \\ 5 United Graduate School of Veterinary Sciences, Yamaguchi University, Yamaguchi, Japan \\ ${ }^{6}$ Saga University, Saga, Japan \\ ${ }^{7}$ Otsuka Pharmaceutical Factory, Tokushima, Japan \\ ${ }^{8}$ Department of Metabolism and Endocrinology, Institute of Clinical Medicine, University of Tsukuba, Tsukuba, Japan
}

\section{Abstract}

Aims/hypothesis. Dysfunctions of lipoprotein lipase (LPL) have been found to be associated with dyslipidaemias, atherosclerosis, obesity and insulin resistance. There are two conflicting hypotheses regarding the roles of LPL in glucose metabolism and insulin resistance. Whether systemically increased LPL activity would be beneficial or detrimental to insulin sensitivity is yet to be resolved. To address this issue, we studied transgenic rabbits overexpressing human LPL transgene.

Methods. LPL transgenic and control rabbits were fed a $10 \%$ high-fat diet (HFD) for 16 weeks. To evaluate glucose metabolism, we compared plasma levels of glucose and insulin in transgenic rabbits with control rabbits and performed an intravenous glucose tolerance test. In addition, we measured adipose tissue accumulation in HFD-fed rabbits.

Results. Increased LPL activity in transgenic rabbits resulted in a significant reduction of plasma triglycerides and non-esterified fatty acids, but not in basal levels of glucose and insulin. HFD feeding induced an elevation of plasma glucose levels accompanied by hyperinsulinaemia in control rabbits, but was significantly inhibited in transgenic rabbits. The intravenous glucose tolerance test showed that transgenic rabbits had faster glucose clearance associated with lower levels of insulin secretion than control rabbits. In addition, there was a significant reduction of body adipose tissue in transgenic rabbits compared with in control rabbits fed an HFD. Scanning electron microscopic examination revealed that adipocytes in transgenic rabbits were predominately small cells.

Conclusions/interpretation. Our results showed that systemically increased LPL activity improves insulin resistance and reduces adipose accumulation in transgenic rabbits, indicating that systemic elevation of LPL may have potential benefits for the treatment of insulin resistance and obesity.

Keywords Diabetes Insulin resistance $\cdot$ Lipoprotein lipase $\cdot$ Obesity $\cdot$ Transgenic rabbits
Received: 11 December 2003 / Accepted: 1 March 2004

Published online: 25 June 2004

(C) Springer-Verlag 2004

S. Kitajima $(\varangle)$

Analytical Research Center for Experimental Sciences,

Saga Medical School, 5-1-1 Nabeshima, Saga 849-8501, Japan

E-mail: kitajims@med.saga-u.ac.jp

Tel.: +81-952-316511 ext. 2433

Abbreviations: ACO, acyl-CoA oxidase - CPT1, carnitinepalmitoyl-transferase- 1 . HFD, high-fat diet - LPL, lipoprotein lipase $\cdot$ MRI, magnetic resonance imaging $\cdot$ PPAR, peroxisome proliferator-activated receptor $\cdot \mathrm{TG}$, triglycerides

\section{Introduction}

Insulin resistance, which can in part be defined as a reduced capacity of insulin to increase glucose uptake and metabolism in target tissues such as skeletal muscle and adipose tissue, has long been associated with elevation of plasma triglycerides (TG) and NEFA [1]. Therefore, understanding the factors that modulate TG and NEFA levels could provide clues for the treatment and prevention of the disease.

Lipoprotein lipase (LPL) plays a pivotal role in lipids and the metabolism of lipoprotein [2, 3]. Major 
functions of LPL include the hydrolysis of TG-rich lipoproteins and the release of NEFA, which are taken up and used for metabolic energy in peripheral tissue such as muscle, or are re-esterified into TG and stored in adipose tissue $[2,3]$. The balance between these competing effects could determine whether increased LPL activity will lead to a reduced rate of weight gain (through greater disposal of ingested fats as metabolic fuel) or to increased adiposity through increased rates of adipose tissue storage of TG. Several previous studies have shown that an imbalance of LPL activity may alter the partitioning of plasma TG between muscle and adipose tissue, and thus influence insulin resistance and obesity. For example, increased LPL in muscle and liver might lead to the over-production of NEFA, to accumulation of TG, and to the subsequent impairment of the insulin signal, resulting in a state of insulin resistance [4]. In addition, LPL activity was found to be higher in visceral adipose tissue of obese mice and humans, which could lead to increased NEFA influx through the portal vein into the liver, and could again result in a state of insulin resistance $[5,6]$. Taken together, these studies suggest that high LPL levels could be associated with, or are the causes of, insulin resistance and obesity.

On the other hand, increased LPL levels have been shown to have completely opposite effects on glucose metabolism and insulin sensitivity. Patients deficient in LPL have hypertriglyceridaemia and high levels of NEFA, which is associated with insulin-resistant diabetes and can be ameliorated by lowering concentrations of TG [7]. Administration of the compound NO-1886, an LPL-promoting agent in rats [8], rabbits [9] and pigs [10] fed a high-fat diet (HFD) suppresses fat accumulation and insulin resistance. Therefore, it is not clear, when within a physiological range, whether "the higher, the better" or "the lower, the better" is a more accurate description in terms of LPL activity in glucose tolerance and adipose tissue. This is an important issue, as ascertaining the true situation will help us to understand whether raising LPL levels can be used as a therapeutic method to treat hyperlipidaemias, diabetes and obesity, or whether it is unsafe to use LPL-raising agents in patients with such conditions $[11,12]$.

It might be difficult to elucidate the multiple and complex roles of LPL in humans in terms of insulin resistance and its related disorders such as diabetes and obesity. One of the best experimental approaches is to use transgenic animals that overexpress LPL. Several lines of transgenic mice that overexpress human LPL have been created, and although the sites of overexpression differ, all transgenic mice show decreased plasma levels of TG $[4,13,14,15,16,17$, 18]. However, studies on transgenic mice have also generated conflicting results regarding the effects of LPL on insulin resistance. For example, two studies on LPL transgenic mice have shown that overexpres- sion of human LPL in mouse muscle is associated with insulin resistance [4, 17], whereas one group (even using the same transgenic mice as in the above study) failed to show a correlative or causal relationship between increased LPL activity and insulin resistance even though there was increased TG content in the muscles [18]. In addition, one study reported that LPL transgenic mice were protected against diet-induced obesity regardless of slight hyperglycaemia [16]. Collectively, these transgenic mouse studies indicate that overexpression of LPL results in (i) reduced plasma levels of TG accompanied by accumulation of TG in muscles, (ii) decreased adipose tissue or diet-induced obesity, and (iii) remaining controversy as to whether or not elevated LPL activity would provide protection from insulin resistance and obesity.

Our laboratory generated transgenic rabbits that systemically overexpress human LPL, in an attempt to investigate the relationship between LPL and atherosclerosis, and reported that increased LPL has a profound impact on lipid metabolism and atherogenesis [19]. Since features of lipoprotein metabolism in rabbits are very similar to those in humans (so-called LDL mammals) but are unlike those of rodents (socalled HDL mammals) [20], we envisioned that LPL transgenic rabbits might be an adequate model for the study of the cause-and-effect relationship between LPL and metabolic disorders such as insulin resistance and obesity. Furthermore, several lines of evidence have shown that even expression of the same transgene in transgenic mice and rabbits leads to completely different interpretations, possibly due to the intrinsic differences between rabbits and rodents [20]. A striking example of such difference between two species is that high expression of LPL in transgenic mice induces myopathies, which is not the case in transgenic rabbits, as reported by our laboratory [21]. In this study, we were especially interested in investigating whether increased LPL activity causes an improvement or a deterioration in glucose metabolism and obesity.

\section{Materials and methods}

Animals. Human-LPL-overexpressing transgenic rabbits were generated by microinjection of human LPL cDNA under the control of the chicken $\beta$-actin promoter [22] as described previously [19]. A male LPL transgenic rabbit (F1 from the L17 line) supplied by Tsukuba University was mated with normal female Japanese White rabbits (JW:kbt; Biotek, Saga, Japan) in our facility. Hemizygous male transgenic rabbits (hLPL gene $^{+/ 0}$ ) and control littermates were used in this study. During the experiment, rabbits were housed individually in metal cages in a room maintained at constant temperature $\left(24 \pm 2{ }^{\circ} \mathrm{C}\right)$ and humidity $(55 \pm 15 \%)$. They were given free access to water. Rabbits were analysed at the age of 16 weeks, having previously been fed a chow diet, and were then fed an HFD composed of $10 \%(\mathrm{w} / \mathrm{w})$ corn oil and lard (corn oil : lard $=2: 1$, by weight) in standard rabbit chow (CRB-1; CLEA, Tokyo, 
Japan) for 16 weeks. At the end of the experiment, rabbits were killed by overdose of thiamylal sodium for the determination of body fat, tissue TG contents and LPL activity. The experimental protocols were approved by the Saga University Animal Experimentation Committee and performed according to the Saga University Guidelines for Animal Experimentation.

Analyses of LPL activity and plasma lipids. After overnight fasting $(16 \mathrm{~h})$, rabbits were injected with a bolus injection of heparin at a dose of $30 \mathrm{U} / \mathrm{kg}$ and blood was collected $10 \mathrm{~min}$ after injection. The enzymatic activity of LPL in post-heparin plasma was determined by the method described previously [19]. Plasma TG, total cholesterol, HDL cholesterol and NEFA were determined using commercially available kits (Wako Pure Chemical Industries, Osaka, Japan).

Intravenous glucose tolerance test. For the evaluation of glucose metabolism, rabbits were fasted overnight and an IVGTT was performed [23]. After rabbits were injected with glucose solution $(0.6 \mathrm{~g} / \mathrm{kg})$, a blood sample was drawn at 5, 10, 15, 20, 30, 45, 60, 75 and $120 \mathrm{~min}$. Plasma glucose and NEFA were assayed using Wako kits. Plasma insulin was determined using a commercial ELISA kit (Morinaga, Yokohama, Japan) with rabbit insulin as standard.

Magnetic resonance imaging analysis. For the evaluation of adipose tissue after HFD feeding, rabbits were scanned using magnetic resonance imaging (MRI). For this analysis, rabbits were anaesthetised with ketamin $(25 \mathrm{mg} / \mathrm{kg}$, i.m.) plus medetomidine $(0.5 \mathrm{mg} / \mathrm{kg}$, s.c. $)$ before scanning to avoid artifacts due to movement. Imaging was performed with $0.2 \mathrm{~T}$ on a wholebody system (MRP20; Hitachi Medical Corporation, Tokyo, Japan) using a knee quadrature coil for humans with a diameter of $15 \mathrm{~cm}$ and length of $18 \mathrm{~cm}$. To reduce artifacts caused by tissue heterogeneities, a three-dimensional spin-echo sequence with a repetition time of $500 \mathrm{~ms}$, an echo time of $25 \mathrm{~ms}$, and a flip angle of $90^{\circ}$ was applied. The investigated volume consisted of 10 cross sections focused on the abdomen with a slice thickness of $6 \mathrm{~mm}$, using a field-of-view of $220 \mathrm{~mm}$, and a scanning matrix of $256 \times 256 \mathrm{~mm}$.

Body fat contents. After 16 weeks of the HFD, adipose tissues from the whole body were carefully removed, weighed while still wet and expressed as a percentage of the body weight. We divided adipose tissues into (i) subcutaneous adipose tissue including fat from the inguinal, axilla, and scapular regions, and (ii) visceral adipose from the abdominal cavity, mesenterium, and retroperitoneal fat.

Cellular size distribution of adipocytes. Adipose tissues were fixed in $10 \%$ neutral-buffered formalin and post-fixed in $1 \%$ osmium tetraoxide. The size of adipocytes in transgenic and control rabbits was examined by scanning electron microscope (JSM-6320F; JEOL, Tokyo, Japan). Each part of the adipose tissues was randomly observed under $\times 200$ magnification and at least ten pictures were taken. The diameter of at least 100 adipocytes was measured using a MacScope image analysis system.

Quantitation of tissue LPL activity and TG contents. After overnight fasting, the liver and skeletal muscle (soleus) were dissected and fixed in $10 \%$ neutral-buffered formalin for histological examination. After fixation, these tissues were embedded in paraffin, cut into sections $(5 \mu \mathrm{m}$ thick) and stained with hematoxylin-eosin. These tissues were also snap-frozen in liquid nitrogen for measurement of TG contents. Lipids in the liver and skeletal muscle were extracted by methods described previously [17, 24], and TG concentrations were measured according to the method by Danno et al. [25]. Tissue LPL activity in liver, adipose and muscle was analysed as previously reported [11].

Northern blot analysis of peroxisome proliferator-activated receptor- $\alpha$ and $\beta$-oxidation-related enzymes. Adipose tissue and liver from rabbits fed HFD were snap-frozen in liquid nitrogen and total RNA was isolated using Trizol reagent. Expression of peroxisome proliferator-activated receptor (PPAR)- $\alpha$, acylCoA oxidase (ACO) and carnitine-palmitoyl-transferase1 (CPT1) in these tissues was evaluated by northern blotting hybridised with ${ }^{32} \mathrm{P}$-labelled mouse cDNA probes.

Statistical analysis. Results were expressed as means \pm SEM. Statistical analysis was performed using either the Student's $t$ test for the data with equal $F$ value or Welch's $t$ test when the $F$ value was not equal. A $p$ value of less than 0.05 was considered statistically significant.

\section{Results}

LPL activity, plasma lipids, glucose and insulin levels. LPL activity of post-heparin plasma in transgenic rabbits was approximately four-fold higher than that of control rabbits (Table 1). Transgenic rabbits had significantly lower levels of plasma TG, total cholesterol and HDL cholesterol than control rabbits on both chow and HFD (Table 2). Analysis of LPL activity of post-heparin plasma of HFD-fed rabbits revealed that HFD feeding resulted in a two-fold increase in LPL activity in transgenic rabbits but 50\% reduction in

Table 1. Plasma LPL activity and concentration of NEFA in transgenic and control rabbits

\begin{tabular}{lll}
\hline & $\begin{array}{l}\text { LPL } \\
\left(\mu \mathrm{mol} \mathrm{NEFA} \cdot \mathrm{ml}^{-1} \cdot \mathrm{min}^{-1}\right)\end{array}$ & $\begin{array}{l}\mathrm{NEFA} \\
(\mathrm{mEq} / \mathrm{l})\end{array}$ \\
\hline Control $(n=6)$ & $0.125 \pm 0.020$ & $0.282 \pm 0.040$ \\
Transgenic $(n=8)$ & $0.525 \pm 0.030^{* *}$ & $0.172 \pm 0.028^{*}$ \\
\hline
\end{tabular}

Data are expressed as means \pm SEM. $\mathrm{mEq}$, microequivalent. All rabbits were aged $20-24$ weeks. $* * p<0.01, * p<0.05$ vs control

Table 2. Plasma lipids in transgenic and control rabbits fed on chow or an HFD

\begin{tabular}{lllll}
\hline & & $\begin{array}{l}\text { TG } \\
(\mathrm{mmol} / \mathrm{l})\end{array}$ & $\begin{array}{l}\mathrm{TC} \\
(\mathrm{mmol} / \mathrm{l})\end{array}$ & $\begin{array}{l}\text { HDL-C } \\
(\mathrm{mmol} / \mathrm{l})\end{array}$ \\
\hline $\begin{array}{l}\text { Control } \\
(n=11-12)\end{array}$ & HFD & $\begin{array}{l}0.37 \pm 0.02 \\
0.56 \pm 0.05 \dagger\end{array}$ & $\begin{array}{l}0.59 \pm 0.05 \\
0.36 \pm 0.06 \dagger\end{array}$ & $\begin{array}{l}0.43 \pm 0.04 \\
0.23 \pm 0.04 \dagger\end{array}$ \\
$\begin{array}{l}\text { Transgenic } \\
(n=9-11)\end{array}$ & Chow & $0.08 \pm 0.01 *$ & $0.34 \pm 0.06^{*}$ & $0.21 \pm 0.05^{*}$ \\
\hline
\end{tabular}

Data are expressed as means \pm SEM. TC, total cholesterol; HDL-C, HDL cholesterol. The comparisons were made between chow diet and HFD in control rabbits ( $\uparrow p<0.01$ vs chow) or between transgenic and control rabbits fed an HFD $(* p<0.01$ vs control) 

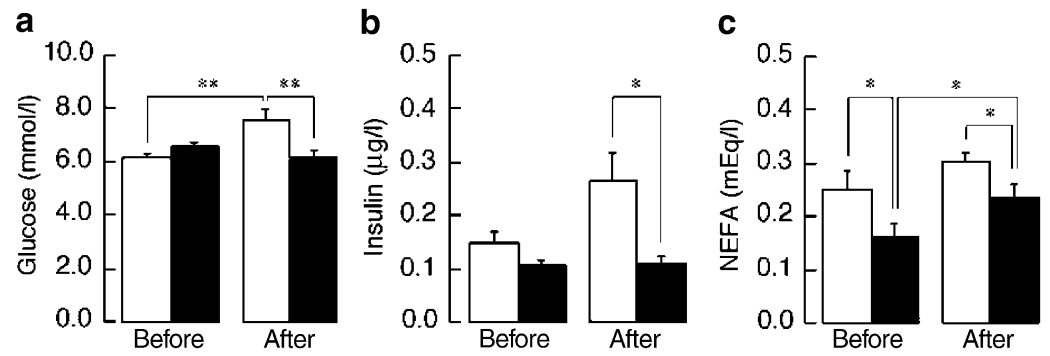

Fig. 1. Plasma glucose (a), insulin (b) and NEFA (c) levels in transgenic (black bars) and control (white bars) rabbits before and after feeding of an HFD for 16 weeks. Data are expressed as means \pm SEM. $n=10$ for each group. ${ }^{*} p<0.05, * * p<0.01$

control rabbits. In this study we focused on the effects of overexpression of LPL on glucose metabolism in transgenic rabbits. On a chow diet, there was no difference in plasma glucose and insulin levels between transgenic and control rabbits, but transgenic rabbits had a lower level of NEFA ( $p<0.05$; Fig. 1a-c).

When fed an HFD, the glucose and insulin levels in control rabbits were significantly increased $(p<0.01)$. However, this HFD-induced effect was significantly inhibited in transgenic rabbits in which glucose and insulin levels remained unchanged (Fig. 1a, b). HFD feeding elevated the plasma level of NEFA in both control and transgenic rabbits, but the degree of NEFA increase was less prominent in transgenic rabbits than in control rabbits (Fig. 1c). Body weights of LPL transgenic and control rabbits at the start of the experiment were simi$\operatorname{lar}(3377.8 \pm 71.6 \mathrm{~g}$ vs $3318.0 \pm 44.1 \mathrm{~g})$. Increase in body weight in transgenic rabbits was slightly lower than that in control rabbits after 16 weeks of the HFD, but we did not find a significant difference between the two groups (842.1 $\pm 96.3 \mathrm{~g}$ vs $986.0 \pm 95.7 \mathrm{~g})$. Daily HFD consumption was not different between transgenic and control rabbits (data not shown).

Intravenous glucose tolerance test. To investigate the effect of increased LPL activity on insulin sensitivity, rabbits on either a chow diet or an HFD were fasted overnight and an IVGTT was performed. Transgenic and control rabbits on a chow diet showed a similar rate of plasma glucose clearance (data not shown). Of the animals on an HFD, transgenic rabbits showed a faster rate of glucose clearance from blood than control rabbits (Fig. 2a). In these transgenic rabbits, insulin and NEFA levels were also constantly lower than those in control rabbits (Fig. 2b, c). The insulin secretion induced by the glucose injection into transgenic rabbits reached a peak at $10 \mathrm{~min}$ and returned to the basal level by $120 \mathrm{~min}$. On the other hand, plasma insulin level in control rabbits was constantly higher than that in transgenic rabbits (Fig. 2b). In transgenic rabbits, plasma NEFA levels remained lower than those in control rabbits at all time-points (Fig. 2c). We found that the AUC of glucose, insulin and NEFA in

transgenic rabbits were significantly lower than those in control rabbits $(p<0.05$; Table 3$)$. The insulin resistance index [26] calculated from the IVGTT in transgenic rabbits was significantly lower than that in control rabbits $(p<0.05$; Table 3$)$.

MRI analysis and body fat contents. After feeding the rabbits an HFD for 16 weeks, we analysed the body fat accumulation by T1-weighted MRI. In T1-weighted MRI analysis, the adipose tissue appears bright in contrast to the other tissue. MRI analysis revealed that the visceral fat mass of transgenic rabbits was remarkably less than that of control rabbits (Fig. 3a). The re- 


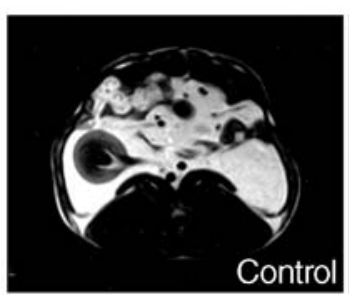

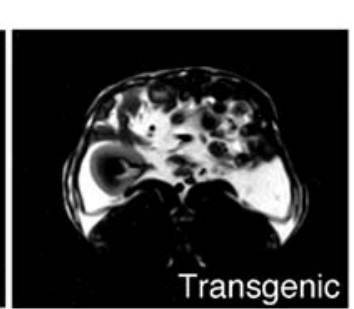

b

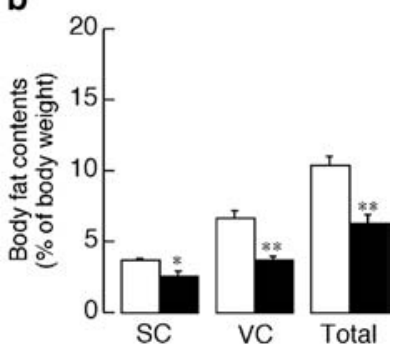

Fig. 3. MRI examination (a) and body fat contents (b) in transgenic (black bars) and control (white bars) rabbits after feeding of an HFD for 16 weeks. SC, subcutaneous fat; VC, visceral fat. Data are expressed as means \pm SEM. $n=10$ for each group. $* p<0.05, * * p<0.01$ a

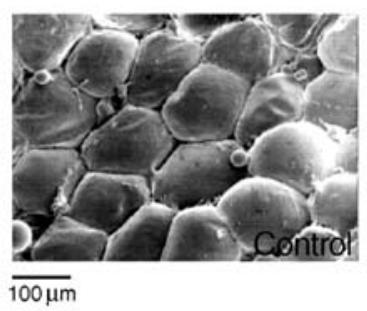

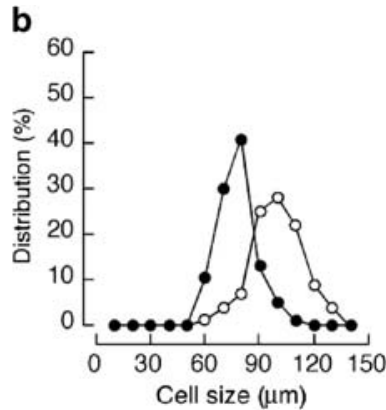

Fig. 4. Micrographs of adipocytes by scanning electron microscope (a) and size distribution of adipocytes (b) in transgenic (black circles) and control (white circles) rabbits on an HFD for 16 weeks

a

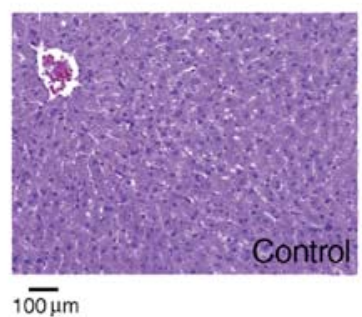
$\overline{100 \mu \mathrm{m}}$

Fig. 5. Histological features of liver (a) and TG contents in the liver and skeletal muscle (b) of transgenic (black bars) and control (white bars) rabbits on an HFD for 16 weeks. Data are b

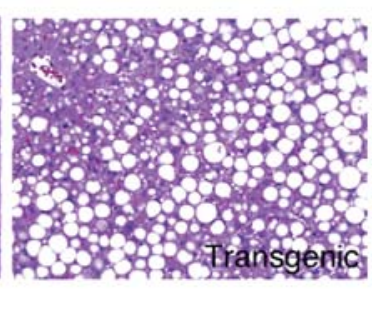

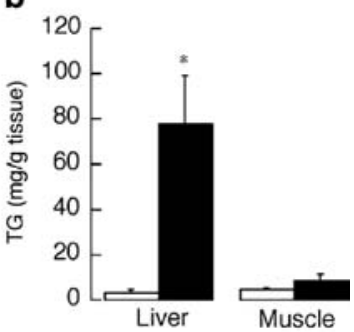

expressed as means \pm SEM. $n=6$ and $n=8$ for transgenic and control rabbits respectively. ${ }^{*} p<0.05$

Table 3. Comparison of AUC of glucose, insulin and NEFA in transgenic and control rabbits during an IVGTT

\begin{tabular}{|c|c|c|c|c|}
\hline & \multicolumn{3}{|l|}{ AUC } & \multirow[t]{2}{*}{ IR index } \\
\hline & Glucose $\left(\mathrm{mmol} \cdot \mathrm{l}^{-1}\right)$ & Insulin $\left(\mu \mathrm{g} \cdot 1^{-1}\right)$ & NEFA $\left(\mathrm{mEq} \cdot 1^{-1}\right)$ & \\
\hline Transgenic $(n=7)$ & $25.3 \pm 1.3^{*}$ & $2.52 \pm 0.42 *$ & $1.226 \pm 0.170 *$ & $0.07 \pm 0.01 *$ \\
\hline
\end{tabular}

Data are expressed as means \pm SEM. mEq, microequivalent. Insulin resistance (IR) index was calculated from product of AUC of glucose and insulin $\times 10^{-3}$ according to the method by Mondon [26]. ${ }^{*} p<0.05$ vs control

duced fat contents were also confirmed by measuring the actual weight of the adipose tissues. In transgenic rabbits, subcutaneous and visceral fat contents were significantly lower than those in control rabbits (Fig. 3b). Scanning electron microscopic examination revealed that adipocytes in transgenic rabbits were predominately small cells when compared with those in control rabbits (Fig. 4). The mean diameters of adipocytes in transgenic and control rabbits were $72.7 \pm 1.0 \mu \mathrm{m}$ and $94.4 \pm 1.4 \mu \mathrm{m}$ respectively.

$T G$ content in the liver and skeletal muscle. Histological examination showed many lipid droplets in the liver of LPL transgenic rabbits (Fig. 5a). TG content in 
Table 4. Tissue LPL activity in transgenic and control rabbits fed an HFD for 16 weeks

\begin{tabular}{llll}
\hline & Skeletal muscle & Liver & Adipose tissue \\
\hline Control $(n=5)$ & $0.057 \pm 0.034$ & $0.254 \pm 0.045$ & $0.031 \pm 0.007$ \\
Transgenic $(n=5)$ & $0.249 \pm 0.061 * *$ & $0.989 \pm 0.124 * *$ & $0.039 \pm 0.006$ \\
\hline
\end{tabular}

Data are expressed as means \pm SEM. Values are given in $\mu$ mol NEFA.g tissue ${ }^{-1} \cdot \mathrm{min}^{-1}$. All rabbits were fed an HFD for 16 weeks. $* * p<0.01$ vs control
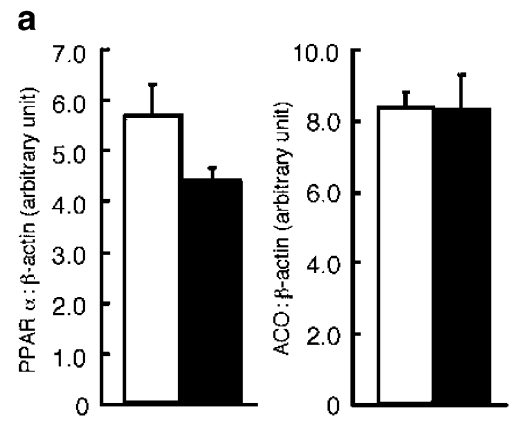

\section{b}

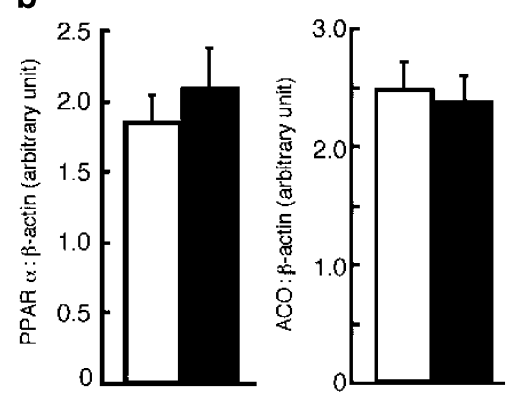

Fig. 6. Northern blot analysis of PPAR- $\alpha$ and ACO gene expressions in liver (a) and adipose tissue (b) of transgenic (black bars) and control (white bars) rabbits after feeding of an HFD for 16 weeks. The expression levels were calculated and expressed as the mean ratio to $\beta$-actin expression \pm SEM. $n=5$ and $n=4$ for transgenic and control rabbits respectively

the liver of transgenic rabbits was 25-fold higher than that in control rabbits $(p<0.05)$, whereas in skeletal muscle, TG content in transgenic rabbits was slightly increased (Fig. 5b). Analysis of tissue LPL activity revealed that transgenic rabbits had a five-fold higher LPL activity in liver and muscle than control rabbits, but adipose tissue LPL activity was not different from that in control rabbits (Table 4). Northern blot analysis showed that expression of PPAR- $\alpha$ and ACO was higher in the liver than in adipose tissue of both transgenic and control rabbits (Fig. 6), whereas CPT1 expression was barely detectable (data not shown). There was no significant difference between the two groups.

\section{Discussion}

In this study, we demonstrated that overexpression of LPL in transgenic rabbits improves HFD-induced insulin resistance and obesity. When challenged with an IVGTT, glucose disposal curve and insulin response in transgenic rabbits were significantly different from controls: they had lower levels of insulin and NEFA associated with decreased AUC values and insulin resistance index. LPL hydrolyses TG-rich lipoproteins and results in the release of NEFA, which are either taken up and used for metabolic energy in peripheral tissue such as muscle and liver or re-esterified into TG and stored in adipose tissue. In IVGTT experiments, NEFA tended to be cleared faster in transgenic rabbits than in control rabbits, possibly due to enhanced LPL-mediated uptake of NEFA in peripheral (non-adipose) tissues. In support of this notion, a recent study has shown that LPL is a key enzyme for the generation of PPAR- $\alpha$ ligands [27], thereby promoting $\beta$-oxidation and ketogenesis. Transgenic rabbits had less fat accumulation and their adipocytes were mainly smaller in size in comparison with those in control rabbits. This phenomenon could be explained by assuming that systemically increased LPL activity decreases fat accumulation by accelerating the lipolytic process or inhibiting de novo lipogenesis, which was caused by competitively increased uptake of NEFA by non-adipose tissue such as muscle, although this hypothesis remains to be verified. Alternatively, LPL may regulate or interact with other proteins that are associated with lipogenesis and/or lipolysis in adipocytes such as hormone-sensitive lipase and PPAR $-\gamma$ or $-\delta$. We are currently investigating these molecular mechanisms. Nevertheless, it is generally believed that smaller adipocyte size is associated with improved insulin sensitivity [28]. If this is the case, a reduced adipocyte size along with lower levels of plasma NEFA in transgenic rabbits may help to explain why transgenic rabbits have increased insulin sensitivity compared with control rabbits when fed an HFD. In support of this notion, it has been reported that small adipocytes can uptake and oxidise more glucose than large adipocytes in the presence of insulin [29]. It is unknown whether small adipocytes secrete humoral factors, such as adiponectins and TNF- $\alpha$, differently from large adipocytes.

Transgenic rabbits had prominent fatty liver on an HFD. The possible mechanism for this "unwanted" side-effect may be attributable to the high level of hepatic LPL expression in transgenic rabbits (Table 4). Increased LPL activity in the liver may lead to the enhanced uptake of TG-rich lipoproteins through receptor- and non-receptor-mediated pathways [19]. Al- 
though we cannot exclude the possibility that transgenic rabbits may have (locally) hepatic insulin resistance associated with the fatty liver [30, 31, 32], overall effect of LPL overexpression on insulin sensitivity was apparently beneficial. These findings in transgenic rabbits contrast with the results in transgenic mice that showed insulin resistance accompanied by accumulation of TG in their skeletal muscle [16] or liver [4]. Nevertheless, one study reported that there is no effect of the accumulation of TG in skeletal muscle on insulin-stimulated whole-body and muscle-specific glucose uptake [18]. Another study [17] found no difference in the muscle TG contents between musclespecific LPL transgenic and control mice, in spite of transgenic mice having a state of insulin resistance. Therefore, it is not clear whether rabbits are different from mice in terms of LPL effects on glucose metabolism and insulin resistance.

In spite of this, our results favour the notion of the elevation of LPL activity, such as by the compound NO-1886 [8, 9, 10, 11] or by LPL gene therapy $[12,33]$, for therapeutic treatments of hypertriglyceridaemia, obesity and related disorders. Compound NO-1886 has been shown to increase LPL mRNA in adipose tissue, myocardium and skeletal muscle, resulting in an elevation of postheparin plasma LPL activity and a decrease in serum TG. Long-term treatment with NO-1886 inhibited the development of atherosclerotic lesions in coronary arteries and aorta in rats [11]. Moreover, NO-1886 treatment suppressed body fat accumulation and improved insulin resistance in rats [8], rabbits [9] and pigs [10], induced by feeding of HFD. Interestingly, NO-1886 also effectively ameliorated fatty liver in obese rats, suggesting that hepatic expression in transgenic rabbits was detrimental whereas increased muscle LPL was beneficial [34].

LPL transgenic rabbits also revealed an interesting and unexpected finding regarding the inhibitory effect of LPL on adipose tissue accumulation. Genetic and diet-induced obesity are associated with high levels of LPL in adipose tissue of humans [35] and rodents [36]. However, these results may only suggest that high LPL in adipose tissue is required to maintain obesity, but is not causative of that obesity. Consistent with findings in LPL-activator-treated rats [8], transgenic rabbits on an HFD had reduced adipose tissue accumulation compared with control rabbits, which has raised an interesting question: does LPL enhance disposal of ingested fats as metabolic fuel, or does it reduce adiposity through decreased rates of adipose tissue storage of TG? In future studies, we hope to examine whether increased LPL in transgenic rabbits leads to increased energy expenditure, such as a decreased respiratory quotient (i.e. increased lipid oxidation), or to increased oxygen consumption.

In conclusion, we have demonstrated that systemically increased LPL activity in transgenic rabbits did not lead to impaired insulin resistance and glucose tolerance. On the contrary, transgenic rabbits had relatively lower levels of insulin and NEFA compared with controls, suggesting that LPL may increase insulin sensitivity of the whole body. Furthermore, the current study indicates that systemic elevation of LPL may protect against HFD-induced insulin resistance and obesity. Although elucidation of the mechanistic underpinnings of these phenomena requires further investigation, these results suggest that LPL might be a therapeutic target for treatments of atherosclerosis as well as insulin resistance and obesity.

Acknowledgements. We thank F. Muto, S. Nakahara, H. Ideguchi, N. Watanabe and A. Higuchi for their technical assistance at the Saga University. We also thank K. Iwata (Otsuka Pharmaceutical Factory) for the analysis of tissue LPL activity and H. Shimano (Institute of Clinical Medicine, University of Tsukuba) for providing us with helpful suggestions and mouse PPAR- $\alpha$, ACO and CTP1 cDNA. This work was supported by Grants-in-Aid for Scientific Research from the Ministry of Education, Science and Culture of Japan $(14570150,15500299)$ and by a grant from the TARA Center of Tsukuba University.

\section{References}

1. Randle PJ, Garland PB, Hales CN, Newsholme EA (1963) The glucose-fatty acid cycle: its role in insulin sensitivity and the metabolic disturbances of diabetes mellitus. Lancet $1: 785-789$

2. Brunzell J (1995) Familial lipoprotein lipase deficiency and other causes of the chylomicronemia syndrome. In: Scriver C, Beandet A, Sly W, Valle D (eds) The metabolic and molecular basis of inherited diseases. McGraw-Hill, New York, pp 1913-1932

3. Goldberg IJ (1996) Lipoprotein lipase and lipolysis: central roles in lipoprotein metabolism and atherogenesis. J Lipid Res 37:693-707

4. Kim JK, Fillmore JJ, Chen Y et al. (2001) Tissue-specific overexpression of lipoprotein lipase causes tissue-specific insulin resistance. Proc Natl Acad Sci USA 8:7522-7752

5. Hotamisligil GS, Shargill NS, Spiegelman BM (1993) Adipose expression of tumor necrosis factor- $\alpha$ : direct role in obesity-linked insulin resistance. Science 259:87-91

6. Hotamisligil GS, Peraldi P, Budavari A, Ellis R, White MF, Spiegelman BM (1996) IRS-1-mediated inhibition of insulin receptor tyrosine kinase activity in TNF-alpha- and obesity-induced insulin resistance. Science 271:665-668

7. Mingrone G, Henriksen FL, Greco AV et al. (1999) Triglyceride-induced diabetes associated with familial lipoprotein lipase deficiency. Diabetes 48:1258-1263

8. Kusunoki M, Hara T, Tsutsumi K et al. (2000) The lipoprotein lipase activator, NO-1886, suppresses fat accumulation and insulin resistance in rats fed a high-fat diet. Diabetologia 43:875-880

9. Yin W, Yuan Z, Tsutsumi K et al. (2003) A lipoprotein lipase-promoting agent, NO-1886, improves glucose and lipid metabolism in high fat, high sucrose-fed New Zealand White rabbits. Exp Diab Res 4:27-34

10. Yin W, Liao D, Wang Z et al. (2004) No-1886 inhibits size of adipocytes, suppresses plasma levels of tumor necrosis factor- $\alpha$ and free fatty acids, improves glucose metabolism in high-fat/high-sucrose-fed miniature pigs. Pharmacol Res 49:199-206 
11. Tsutsumi K, Inoue Y, Shima A, Iwasaki K, Kawamura M, Murase T (1993) The novel compound NO-1886 increases lipoprotein lipase activity with resulting elevation of high density lipoprotein cholesterol, and long-term administration inhibits atherogenesis in the coronary arteries of rats with experimental atherosclerosis. J Clin Invest 92:411-417

12. Zsigmond E, Kobayashi K, Tzung KW, Li L, Fuke Y, Chan L (1997) Adenovirus-mediated gene transfer of human lipoprotein lipase ameliorates the hyperlipidemias associated with apolipoprotein $\mathrm{E}$ and $\mathrm{LDL}$ receptor deficiencies in mice. Hum Gene Ther 8:1921-1933

13. Shimada M, Shimano H, Gotoda T et al. (1993) Overexpression of human lipoprotein lipase in transgenic mice. Resistance to diet-induced hypertriglyceridemia and hypercholesterolemia. J Biol Chem 268:17924-17929

14. Liu MS, Jirik FR, LeBoeuf TC et al. (1994) Alteration of lipid profiles in plasma of transgenic mice expressing human lipoprotein lipase. J Biol Chem 269:11417-11424

15. Levak-Frank S, Radner H, Walsh A et al. (1995) Musclespecific overexpression of lipoprotein lipase causes a severe myopathy characterized by proliferation of mitochondria and peroxisomes in transgenic mice. J Clin Invest 96:976-986

16. Jensen DR, Schlaepfer IR, Morin CL et al. (1997) Preventation diet-induced obesity in transgenic mice overexpressing skeletal muscle lipoprotein lipase. Am J Physiol 273:R683-R689

17. Ferreira LDMC-B, Pulawa LK, Jensen DR, Eckel RH (2001) Overexpressing human lipoprotein lipase in mouse skeletal muscle is associated with insulin resistance. Diabetes 50:1064-1068

18. Voshol PJ, Jong MC, Dahlmans VEH et al. (2001) In muscle-specific lipoprotein lipase-overexpressing mice, muscle triglyceride content is increased without inhibition of insulin-stimulated whole-body and muscle-specific glucose uptake. Diabetes 50:2585-2590

19. Fan J, Unoki H, Kojima N et al. (2001) Overexpression of lipoprotein lipase in transgenic rabbits inhibits diet-induced hypercholesterolemia and atherosclerosis. J Biol Chem 43:40071-40079

20. Fan J, Watanabe T (2003) Transgenic rabbits as a therapeutic protein bioreactor and human disease models. Pharmacol Therapeut 99:261-282

21. Koike T, Wang X, Unoki H et al. (2002) Increased expression of lipoprotein lipase in transgenic rabbits does not lead to abnormalities in skeletal and heart muscles. Muscle Nerve 26:823-827

22. Niwa H, Yamamura K, Miyazaki J (1991) Efficient selection for high-expression transfectants with a novel eukaryotic vector. Gene 108:193-199
23. Zhang B, Saku K, Hirata K et al. (1994) Quantitative characterization of insulin-glucose response in Watanabe heritable hyperlipidemic and cholesterol-fed rabbits and the effect of cilazapril. Metabolism 43:360-366

24. Bligh FG, Dyer WJ (1959) A rapid method of total lipid extraction and purification. Can J Biochem Physiol 37:911-917

25. Danno H, Jincho Y, Budiyanto S, Furukawa Y, Kimura S (1992) A simple enzymatic quantitative analysis of triglycerides in tissues. J Nutr Sci Vitaminol 38:517-521

26. Mondon CE, Dolkas CB, Oyama J (1981) Enhanced skeletal muscle insulin sensitivity in year-old rats adapted to hypergravity. Am J Physiol 240:E482-E488

27. Ziouzenkova O, Perrey S, Asatryan L et al. (2003) Lipolysis of triglyceride-rich lipoproteins generates PPAR ligands: evidence for an antiinflammatory role for lipoprotein lipase. Proc Natl Acad Sci USA 100:2730-2735

28. Okuno A, Tamemoto H, Tobe K et al. (1998) Troglitazone increases the number of small adipocytes without the change of white adipose tissue mass in obese Zucker rats. J Clin Invest 101:1354-1361

29. Olefsky JM (1976) The effect of spontaneous obesity on insulin binding, glucose transport, and glucose oxidation of isolated rat adipocyte. J Clin Invest 57:842-851

30. Phillips DIW, Caddy S, Ilic V et al. (1996) Intramuscular triglycerides and muscle insulin sensitivity: evidence for a relationship in non-diabetic subject. Metabolism 45:947950

31. Banerji MA, Buckey MC, Chaiken RL, Gordon D, Lebovitz HE, Kral JG (1995) Liver fat, serum triglycerides and visceral adipose tissue in insulin-sensitive and insulinresistant black men with NIDDM. Int Natl J Obes 19:846-850

32. Koyama K, Chen G, Lee Y, Unger RH (1997) Tissue triglycerides, insulin resistance, and insulin production: implications for hyperinsulinemia of obesity. Am J Physiol 273:E708-E713

33. Schlaepfer IR, Eckel RH (1999) Plasma triglyceride reduction in mice after direct injection of muscle-specific lipoprotein lipase DNA. Diabetes 48:223-227

34. Kusunoki M, Tsutsumi K, Inoue Y et al. (2004) Lipoprotein lipase activator NO-1886 improves fatty liver caused by high-fat feeding in streptozotocin-induced diabetic rats. Metabolism 53:260-263

35. Kern PA, Ong JM, Saffari B, Carty J (1990) The effects of weight loss on the activity and expression of adipose-tissue lipoprotein lipase in very obese humans. N Engl J Med 322:1053-1059

36. Bessesen DH, Robertson AD, Eckel RH (1991) Weight reduction increases adipose but decreases cardiac LPL in reduced-obese Zucker rats. Am J Physiol 261:E246-E251 